\title{
ROBUST PRECONDITIONING FOR STOCHASTIC GALERKIN FORMULATIONS OF PARAMETER-DEPENDENT NEARLY INCOMPRESSIBLE ELASTICITY EQUATIONS*
}

\author{
ARBAZ KHAN ${ }^{\dagger}$, CATHERINE E. POWELL $^{\ddagger}$, AND DAVID J. SILVESTER ${ }^{\S}$
}

\begin{abstract}
We consider the nearly incompressible linear elasticity problem with an uncertain spatially varying Young's modulus. The uncertainty is modelled with a finite set of parameters with prescribed probability distribution. We introduce a novel three-field mixed variational formulation of the PDE model and discuss its approximation by stochastic Galerkin mixed finite element techniques. First, we establish the well-posedness of the proposed variational formulation and the associated finite-dimensional approximation. Second, we focus on the efficient solution of the associated large and indefinite linear system of equations. A new preconditioner is introduced for use with the minimal residual method (MINRES). Eigenvalue bounds for the preconditioned system are established and shown to be independent of the discretisation parameters and the Poisson ratio. The S-IFISS software used for computation is available online.
\end{abstract}

Key words. uncertain material parameters, linear elasticity, mixed approximation, stochastic Galerkin finite element method, preconditioning.

AMS subject classifications. 65N30, 65F08, 35R60.

1. Introduction. The locking of finite element approximations when solving nearly incompressible elasticity problems is a significant issue in the computational engineering world. The standard way of preventing locking is to write the underlying equations as a system and introduce pressure as an additional unknown $[10,11]$. Thus, the starting point for this work is the Herrmann formulation of linear elasticity

$$
\begin{aligned}
-\nabla \cdot \boldsymbol{\sigma} & =\boldsymbol{f} \quad \text { in } D, \\
\nabla \cdot \boldsymbol{u}+\frac{p}{\lambda} & =0, \quad \text { in } D,
\end{aligned}
$$

where $D$ is a bounded Lipschitz polygon in $\mathbb{R}^{2}$ (polyhedral in $\mathbb{R}^{3}$ ). In this setting, the elastic deformation of the isotropic solid is defined in terms of the stress tensor $\boldsymbol{\sigma}$, the body force $\boldsymbol{f}$, the displacement field $\boldsymbol{u}$ and the Herrmann pressure $p$ (auxiliary variable). The stress tensor is related to the strain tensor $\varepsilon$ through the identities

$$
\boldsymbol{\sigma}=2 \mu \varepsilon-p \boldsymbol{I}, \quad \varepsilon=\frac{1}{2}\left(\nabla \boldsymbol{u}+(\nabla \boldsymbol{u})^{\top}\right) .
$$

The Lamé coefficients $\mu$ and $\lambda$ satisfy $0<\mu_{1}<\mu<\mu_{2}<\infty$ and $0<\lambda<\infty$ and can be defined in terms of the Young's modulus $E$ and the Poisson ratio $\nu$ via

$$
\mu=\frac{E}{2(1+\nu)}, \quad \lambda=\frac{E \nu}{(1+\nu)(1-2 \nu)} .
$$

Our focus is on uncertainty quantification. Specifically, we consider the case where the properties of the elastic material are varying spatially in an uncertain way. For

*This work was supported by EPSRC grant EP/P013317. The authors would also like to thank the Isaac Newton Institute for Mathematical Sciences, Cambridge, for support and hospitality during the Uncertainty Quantification programme where work on this paper was partially undertaken. This programme was supported by EPSRC grant EP/K032208/1.

$\dagger$ School of Mathematics, University of Manchester, UK (arbaz.khan@manchester.ac.uk)

$\ddagger$ School of Mathematics, University of Manchester, UK (c.powell@manchester.ac.uk)

$\S$ School of Mathematics, University of Manchester, UK (d.silvester@manchester.ac.uk). 
example, this may be due to material imperfections or inaccurate measurements. To account for this uncertainty we model the Young's modulus $E$ as a spatially varying random field. More precisely, we introduce a vector $\boldsymbol{y}=\left(y_{1}, \ldots, y_{M}\right)$ of parameters, with each $y_{k} \in \Gamma_{k}=[-1,1]$ and represent $E$ as an affine combination

$$
E(\boldsymbol{x}, \boldsymbol{y}):=e_{0}(\boldsymbol{x})+\sum_{k=1}^{M} e_{k}(\boldsymbol{x}) y_{k}, \quad \boldsymbol{x} \in D, \boldsymbol{y} \in \Gamma,
$$

where $\Gamma=\Gamma_{1} \times \cdots \times \Gamma_{M} \subset \mathbb{R}^{M}$ is our parameter domain. Such representations arise, for example, from truncated Karhunen-Loève expansions of second-order random fields. In (1.2), $e_{0}(\boldsymbol{x})$ typically represents the mean, and $e_{k}(\boldsymbol{x}) y_{k}$ is a perturbation away from the mean. The resulting parameter-dependent problem is given by

$$
\begin{aligned}
-\nabla \cdot \boldsymbol{\sigma}(\boldsymbol{x}, \boldsymbol{y}) & =\boldsymbol{f}(\boldsymbol{x}), & & \boldsymbol{x} \in D, \boldsymbol{y} \in \Gamma, \\
\nabla \cdot \boldsymbol{u}(\boldsymbol{x}, \boldsymbol{y})+\frac{p(\boldsymbol{x}, \boldsymbol{y})}{\lambda(\boldsymbol{x}, \boldsymbol{y})} & =0, & & \boldsymbol{x} \in D, \boldsymbol{y} \in \Gamma, \\
\boldsymbol{u}(\boldsymbol{x}, \boldsymbol{y}) & =\boldsymbol{g}(\boldsymbol{x}), & & \boldsymbol{x} \in \partial D_{D}, \boldsymbol{y} \in \Gamma, \\
\boldsymbol{\sigma}(\boldsymbol{x}, \boldsymbol{y}) \boldsymbol{n} & =\mathbf{0}, & & \boldsymbol{x} \in \partial D_{N}, \boldsymbol{y} \in \Gamma,
\end{aligned}
$$

where the boundary of the spatial domain is $\partial D=\partial D_{D} \cup \partial D_{N}$ with $\partial D_{D} \cap \partial D_{N}=\emptyset$ and $\partial D_{D}, \partial D_{N} \neq \emptyset$, the stress tensor is $\sigma: D \times \Gamma \rightarrow \mathbb{R}^{d \times d}(d=2,3)$, the strain tensor is $\varepsilon: D \times \Gamma \rightarrow \mathbb{R}^{d \times d}$, the body force is $f: D \rightarrow \mathbb{R}^{d}$, the displacement field is $\boldsymbol{u}: D \times \Gamma \rightarrow \mathbb{R}^{d}$ and the Herrmann pressure is $p: D \times \Gamma \rightarrow \mathbb{R}$. The Lamé coefficients are also parameter-dependent and spatially varying

$$
\mu(\boldsymbol{x}, \boldsymbol{y})=\frac{E(\boldsymbol{x}, \boldsymbol{y})}{2(1+\nu)}, \quad \lambda(\boldsymbol{x}, \boldsymbol{y})=\frac{E(\boldsymbol{x}, \boldsymbol{y}) \nu}{(1+\nu)(1-2 \nu)} .
$$

Note that we assume that $\nu$ is a given fixed constant and that $0<\mu_{1}<\mu<\mu_{2}<\infty$ and $0<\lambda<\infty$ a.e. in $D \times \Gamma$.

Stochastic Galerkin finite element methods (SGFEMs) are a popular way of approximating solutions to parameter-dependent PDEs. Broadly speaking, we seek approximate solutions in tensor product spaces of the form $X_{h} \otimes S_{\Lambda}$ where $X_{h}$ is an appropriate finite element space associated with a subdivision of $D$ and $S_{\Lambda}$ is, typically, a set of multivariate polynomials that are globally defined on the parameter domain $\Gamma$. This is a feasible strategy if the number of input parameters is modest, and the underlying solution is sufficiently smooth as a function of those parameters. We refer to Babuška et al. [1] for a priori error estimates for SGFEM approximations of solutions to elliptic PDEs with parameter-dependent coefficients and Bespalov et al. [2] for a priori error estimates for SGFEM approximations of solutions to mixed formulations of elliptic PDEs with parameter-dependent coefficients. A posteriori error analysis of linear elasticity with parameter-dependent coefficients is considered by Eigel et al. [5]. Crucial to the efficient implementation of SGFEMs is the need to separate the terms that depend on $\boldsymbol{x}$ from the terms that depend on $\boldsymbol{y}$ in the weak formulation of the problem. Here, since both $\mu$ and $1 / \lambda$ appear in the PDE model (1.3), both $E$ and $E^{-1}$ appear in the formulation.

To address this difficulty, our idea here is to introduce a second auxiliary variable $\tilde{p}=p / E$ to give a distinctive three-field mixed formulation of (1.3): find $\boldsymbol{u}: D \times \Gamma \rightarrow$ 
$\mathbb{R}^{d}$ and $p, \tilde{p}: D \times \Gamma \rightarrow \mathbb{R}$ such that,

$$
\begin{aligned}
-\nabla \cdot \boldsymbol{\sigma}(\boldsymbol{x}, \boldsymbol{y}) & =\boldsymbol{f}(\boldsymbol{x}), & & \boldsymbol{x} \in D, \boldsymbol{y} \in \Gamma, \\
\nabla \cdot \boldsymbol{u}(\boldsymbol{x}, \boldsymbol{y})+\tilde{\lambda}^{-1} \tilde{p}(\boldsymbol{x}, \boldsymbol{y}) & =0, & & \boldsymbol{x} \in D, \boldsymbol{y} \in \Gamma, \\
\tilde{\lambda}^{-1} p(\boldsymbol{x}, \boldsymbol{y})-\tilde{\lambda}^{-1} E(\boldsymbol{x}, \boldsymbol{y}) \tilde{p}(\boldsymbol{x}, \boldsymbol{y}) & =0, & & \boldsymbol{x} \in D, \boldsymbol{y} \in \Gamma, \\
\boldsymbol{u}(\boldsymbol{x}, \boldsymbol{y}) & =\boldsymbol{g}(\boldsymbol{x}), & & \boldsymbol{x} \in \partial D_{D}, \boldsymbol{y} \in \Gamma, \\
\boldsymbol{\sigma}(\boldsymbol{x}, \boldsymbol{y}) \boldsymbol{n} & =\mathbf{0}, & & \boldsymbol{x} \in \partial D_{N}, \boldsymbol{y} \in \Gamma,
\end{aligned}
$$

where

$$
\tilde{\lambda}=\frac{\lambda(\boldsymbol{x}, \boldsymbol{y})}{E(\boldsymbol{x}, \boldsymbol{y})}=\frac{\nu}{(1+\nu)(1-2 \nu)},
$$

is now a fixed constant. The advantage of (1.4) is that while $E$ appears in the first and third equations, $E^{-1}$ does not appear at all. As a result, the discrete problem associated with our SGFEM approximation has a structure that is relatively easy to exploit. This is a novel solution strategy and gives this work a distinctive edge.

The rest of the paper is organised as follows. Section 2 introduces a weak formulation of (1.4) and discusses well posedness. In particular, a stability result is established with respect to a coefficient-dependent norm that is a generalisation of the natural norm identified in our earlier work [11]. Section 3 introduces the finitedimensional problem associated with SGFEM approximation and gives details of the associated linear algebra system that needs to be solved when computing the Galerkin solution. A novel preconditioner is introduced in Section 4 and bounds for the eigenvalues of the preconditioned system are established. The preconditioning strategy is consistent with the philosophy of Mardal and Winther [13]: the diagonal blocks of the preconditioning matrix are associated with the norm for which the stability of the mixed approximation has been established. Finally, we present numerical results in Section 5 to illustrate the efficiency and robustness when representative discrete problems are solved using the minimal residual method.

2. Weak formulation. First, we need to impose some conditions on the model inputs and define appropriate solution spaces. Recall that $E$ is defined as in (1.2) where $\Gamma=\Gamma_{1} \times \cdots \times \Gamma_{M} \subset \mathbb{R}^{M}$ is the parameter domain, and $\Gamma_{k}=[-1,1]$.

Assumption 2.1. The random field $E \in L^{\infty}(D \times \Gamma)$ is uniformly bounded away from zero, i.e., there exist positive constants $E_{\min }$ and $E_{\max }$ such that

$$
0<E_{\min } \leq E(\boldsymbol{x}, \boldsymbol{y}) \leq E_{\max }<\infty \quad \text { a.e. in } D \times \Gamma .
$$

To identify the lower bound, it will be convenient to further assume that

$$
0<e_{0}^{\min } \leq e_{0}(\boldsymbol{x}) \leq e_{0}^{\max }<\infty \quad \text { a.e. in } D \quad \text { and } \quad \frac{1}{e_{0}^{\min }} \sum_{k=1}^{M}\left\|e_{k}\right\|_{L^{\infty}(D)}<1 .
$$

Let $\pi(\boldsymbol{y})$ be a product measure with $\pi(\boldsymbol{y}):=\Pi_{k=1}^{M} \pi_{k}\left(y_{k}\right)$, where $\pi_{k}$ denotes a measure on $\left(\Gamma_{k}, \mathcal{B}\left(\Gamma_{k}\right)\right)$ and $\mathcal{B}\left(\Gamma_{k}\right)$ is the Borel $\sigma$-algebra on $\Gamma_{k}$. We will assume that the parameters $y_{k}$ in (1.2) are images of independent mean zero uniform random variables on $[-1,1]$ and choose $\pi_{k}$ to be the associated probability measure. Now we can define the following Bochner space,

$$
L_{\pi}^{2}(\Gamma, X(D)):=\left\{v(\boldsymbol{x}, \boldsymbol{y}): D \times \Gamma \rightarrow \mathbb{R} ;\|v\|_{L_{\pi}^{2}(\Gamma, X(D))}<\infty\right\},
$$


where $X(D)$ is a normed vector space of real-valued functions on $D$ with norm $\|\cdot\|_{X}$ and

$$
\|\cdot\|_{L_{\pi}^{2}(\Gamma, X(D))}:=\left(\int_{\Gamma}\|\cdot\|_{X}^{2} d \pi(\boldsymbol{y})\right)^{1 / 2} .
$$

In our analysis, we will need the following spaces

$$
\mathcal{V}:=L_{\pi}^{2}\left(\Gamma, \boldsymbol{H}_{E_{0}}^{1}(D)\right), \quad \mathcal{W}:=L_{\pi}^{2}\left(\Gamma, L^{2}(D)\right) \quad \text { and } \quad \mathcal{W}:=L_{\pi}^{2}\left(\Gamma, \boldsymbol{L}^{2}(D)\right),
$$

where $\boldsymbol{H}_{E_{0}}^{1}(D)=\left\{\boldsymbol{v} \in \boldsymbol{H}^{1}(D),\left.\boldsymbol{v}\right|_{\partial D_{D}}=\mathbf{0}\right\}$ and $\boldsymbol{H}^{1}(D)=\boldsymbol{H}^{1}\left(D ; \mathbb{R}^{d}\right)$ is the usual vector-valued Sobolev space with associated norm $\|\cdot\|_{1}$. We assume that the load function satisfies $\boldsymbol{f} \in\left(L^{2}(D)\right)^{d}$ and for simplicity, we choose $\boldsymbol{g}=\mathbf{0}$ on $\partial D_{D}$. In that case, the weak formulation of (1.4) is: find $(\boldsymbol{u}, p, \tilde{p}) \in \mathcal{V} \times \mathcal{W} \times \mathcal{W}$ such that

$$
\begin{aligned}
a(\boldsymbol{u}, \boldsymbol{v})+b(\boldsymbol{v}, p) & =f(\boldsymbol{v}) & & \forall \boldsymbol{v} \in \mathcal{V}, \\
b(\boldsymbol{u}, q)-c(\tilde{p}, q) & =0 & & \forall q \in \mathcal{W}, \\
-c(p, \tilde{q})+d(\tilde{p}, \tilde{q}) & =0 & & \forall \tilde{q} \in \mathcal{W} .
\end{aligned}
$$

Here, we have

$$
\begin{aligned}
a(\boldsymbol{u}, \boldsymbol{v}) & :=\alpha \int_{\Gamma} \int_{D} E(\boldsymbol{x}, \boldsymbol{y}) \varepsilon(\boldsymbol{u}(\boldsymbol{x}, \boldsymbol{y})): \varepsilon(\boldsymbol{v}(\boldsymbol{x}, \boldsymbol{y})) d \boldsymbol{x} d \pi(\boldsymbol{y}), \\
b(\boldsymbol{v}, p) & :=-\int_{\Gamma} \int_{D} p(\boldsymbol{x}, \boldsymbol{y}) \operatorname{div} \boldsymbol{v}(\boldsymbol{x}, \boldsymbol{y}) d \boldsymbol{x} d \pi(\boldsymbol{y}), \\
c(p, q) & :=(\alpha \beta)^{-1} \int_{\Gamma} \int_{D} p(\boldsymbol{x}, \boldsymbol{y}) q(\boldsymbol{x}, \boldsymbol{y}) d \boldsymbol{x} d \pi(\boldsymbol{y}), \\
d(p, q) & :=(\alpha \beta)^{-1} \int_{\Gamma} \int_{D} E(\boldsymbol{x}, \boldsymbol{y}) p(\boldsymbol{x}, \boldsymbol{y}) q(\boldsymbol{x}, \boldsymbol{y}) d \boldsymbol{x} d \pi(\boldsymbol{y}), \\
f(\boldsymbol{v}) & :=\int_{\Gamma} \int_{D} f(\boldsymbol{x}) \boldsymbol{v}(\boldsymbol{x}, \boldsymbol{y}) d \boldsymbol{x} d \pi(\boldsymbol{y}),
\end{aligned}
$$

with

$$
\alpha:=\frac{1}{1+\nu}, \quad \beta:=\frac{\nu}{(1-2 \nu)} .
$$

Note that $\alpha$ and $\beta$ depend on the Poisson ratio $\nu$ but are fixed constants. Following convention, we will also define the bilinear form

$$
\mathcal{B}(\boldsymbol{u}, p, \tilde{p} ; \boldsymbol{v}, q, \tilde{q})=a(\boldsymbol{u}, \boldsymbol{v})+b(\boldsymbol{v}, p)+b(\boldsymbol{u}, q)-c(\tilde{p}, q)-c(p, \tilde{q})+d(\tilde{p}, \tilde{q}),
$$

so as to express (2.4) in the compact form: find $(\boldsymbol{u}, p, \tilde{p}) \in \mathcal{V} \times \mathcal{W} \times \mathcal{W}$ such that

$$
\mathcal{B}(\boldsymbol{u}, p, \tilde{p} ; \boldsymbol{v}, q, \tilde{q})=f(\boldsymbol{v}), \quad \forall(\boldsymbol{v}, q, \tilde{q}) \in \mathcal{V} \times \mathcal{W} \times \mathcal{W} .
$$

The next result establishes that the four bilinear forms appearing in (2.4) and hence the bilinear form $\mathcal{B}(\cdot, \cdot)$ in $(2.12)$, are bounded.

Lemma 2.1. If E satisfies Assumption 2.1, then the following bounds hold

$$
\begin{array}{rlrl}
a(\boldsymbol{u}, \boldsymbol{v}) & \leq \alpha E_{\max }\|\nabla \boldsymbol{u}\|_{\mathcal{W}}\|\nabla \boldsymbol{v}\|_{\mathcal{W}} & & \forall \boldsymbol{u}, \boldsymbol{v} \in \mathcal{V}, \\
b(\boldsymbol{u}, p) \leq \sqrt{d}\|\nabla \boldsymbol{u}\|_{\mathcal{W}}\|p\|_{\mathcal{W}} & & \forall \boldsymbol{u} \in \mathcal{V}, \forall p \in \mathcal{W}, \\
c(p, q) & \leq(\alpha \beta)^{-1}\|p\|_{\mathcal{W}}\left\|_{q}\right\|_{\mathcal{W}} & & \forall p, q \in \mathcal{W}, \\
d(p, q) & \leq(\alpha \beta)^{-1} E_{\max }\|p\|_{\mathcal{W}}\left\|_{q}\right\|_{\mathcal{W}} & & \forall p, q \in \mathcal{W} .
\end{array}
$$


Proof. All bounds follow from the Cauchy-Schwarz inequality and (2.1).

The next result establishes that three of the bilinear forms appearing in (2.4) and (2.12) are coercive and that an inf-sup condition involving $b(\cdot, \cdot)$ is satisfied.

Lemma 2.2. If Assumption 2.1 is valid then the following bounds hold

$$
\begin{aligned}
a(\boldsymbol{u}, \boldsymbol{u}) & \geq \alpha E_{\min } C_{K}\|\nabla \boldsymbol{u}\|_{\mathcal{W}}^{2} & & \forall \boldsymbol{u} \in \mathcal{V}, \\
c(p, p) & \geq(\alpha \beta)^{-1}\|p\|_{\mathcal{W}}^{2} & & \forall p \in \mathcal{W}, \\
d(p, p) & \geq(\alpha \beta)^{-1} E_{\min }\|p\|_{\mathcal{W}}^{2} & & \forall p \in \mathcal{W},
\end{aligned}
$$

where $0<C_{K} \leq 1$ is the Korn constant. In addition, there exists an inf-sup constant $C_{D}>0$ such that

$$
\sup _{0 \neq \boldsymbol{v} \in \mathcal{V}} \frac{b(\boldsymbol{v}, q)}{\|\nabla \boldsymbol{v}\|_{\mathcal{W}}} \geq C_{D}\|q\|_{\mathcal{W}} \quad \forall q \in \mathcal{W}
$$

Proof. The first bound follows by combining (2.1) with Korn's inequality. The second and third bounds follow directly from the definition of the bilinear forms and (2.1). We can use arguments such as in [4, Lemma 11.2.3], [2, Lemma 7.2] (and references therein for non-convex domains $D$ ) as well as [8, Section 4.1.4] (for more general boundary conditions) to show that for any $q \in \mathcal{W}$ there exists a $\boldsymbol{w} \in \mathcal{V}$ such that $\operatorname{div} \boldsymbol{w}=q$ and $C_{D}\|\nabla \boldsymbol{w}\|_{\mathcal{W}} \leq\|q\|_{\mathcal{W}}$, where $C_{D}$ is positive constant. Thus

$$
\sup _{0 \neq \boldsymbol{v} \in \mathcal{V}} \frac{b(\boldsymbol{v}, q)}{\|\nabla \boldsymbol{v}\|_{\mathcal{W}}} \geq \frac{-b(\boldsymbol{w}, q)}{\|\nabla \boldsymbol{w}\|_{\mathcal{W}}}=\frac{\|q\|_{\mathcal{W}}^{2}}{\|\nabla \boldsymbol{w}\|_{\mathcal{W}}} \geq C_{D}\|q\|_{\mathcal{W}}
$$

To establish that our problem formulation is well posed, we now introduce a coefficient-dependent norm $\||\cdot|\|$ on $\mathcal{V} \times \mathcal{W} \times \mathcal{W}$, defined by

$$
\|(\boldsymbol{v}, q, \tilde{q}) \mid\|^{2}:=\alpha\|\nabla \boldsymbol{v}\|_{\mathcal{W}}^{2}+\left(\alpha^{-1}+(\alpha \beta)^{-1}\right)\|q\|_{\mathcal{W}}^{2}+(\alpha \beta)^{-1}\|\tilde{q}\|_{\mathcal{W}}^{2} .
$$

The well-posedness of (2.12) is addressed in the next two results.

Lemma 2.3. If Assumption 2.1 is valid then for any $(\boldsymbol{u}, p, \tilde{p}) \in \mathcal{V} \times \mathcal{W} \times \mathcal{W}$, there exists $(\boldsymbol{v}, q, \tilde{q}) \in \mathcal{V} \times \mathcal{W} \times \mathcal{W}$ with $\left\|\left|(\boldsymbol{v}, q, \tilde{q})\left\|\left|\leq C_{2}\||(\boldsymbol{u}, p, \tilde{p}) \||\right.\right.\right.\right.$, satisfying

$$
\mathcal{B}(\boldsymbol{u}, p, \tilde{p} ; \boldsymbol{v}, q, \tilde{q}) \geq E_{\min } C_{1}\|\mid(\boldsymbol{u}, p, \tilde{p})\| \|^{2},
$$

where $C_{1}$ and $C_{2}$ depend on $E_{\max }, C_{K}$ and $C_{D}$.

Proof. From (2.12) we have

$$
\begin{aligned}
\mathcal{B}(\boldsymbol{u}, p, \tilde{p} ; \boldsymbol{u},-p, \tilde{p}) & =a(\boldsymbol{u}, \boldsymbol{u})+b(\boldsymbol{u}, p)+b(\boldsymbol{u},-p)-c(\tilde{p},-p)-c(p, \tilde{p})+d(\tilde{p}, \tilde{p}), \\
& =a(\boldsymbol{u}, \boldsymbol{u})+d(\tilde{p}, \tilde{p})=:|\boldsymbol{u}|_{a}^{2}+|\tilde{p}|_{d}^{2}
\end{aligned}
$$

Now, as a consequence of (2.20), since $p \in \mathcal{W}$, there exists a $\boldsymbol{w} \in \mathcal{V}$ such that

$$
-b(\boldsymbol{w}, p) \geq C_{D} \alpha^{-1}|| p\left\|_{\mathcal{W}}^{2}, \quad \alpha^{1 / 2}\right\| \nabla \boldsymbol{w}\left\|_{\mathcal{W}} \leq \alpha^{-1 / 2}\right\| p \|_{\mathcal{W}}
$$


Using this particular $\boldsymbol{w}$ in (2.11) and using Lemma 2.1, it follows that

$$
\begin{aligned}
\mathcal{B}(\boldsymbol{u}, p, \tilde{p} ;-\boldsymbol{w}, 0,0) & =-b(\boldsymbol{w}, p)-a(\boldsymbol{u}, \boldsymbol{w}) \\
& \geq C_{D} \alpha^{-1}\|p\|_{\mathcal{W}}^{2}-|\boldsymbol{u}|_{a}|\boldsymbol{w}|_{a} \\
& \geq C_{D} \alpha^{-1}\|p\|_{\mathcal{W}}^{2}-|\boldsymbol{u}|_{a} E_{\max }^{1 / 2} \alpha^{1 / 2}\|\nabla \boldsymbol{w}\|_{\mathcal{W}} \\
& \geq C_{D} \alpha^{-1}\|p\|_{\mathcal{W}}^{2}-|\boldsymbol{u}|_{a} E_{\max }^{1 / 2} \alpha^{-1 / 2}\|p\|_{\mathcal{W}} \\
& \geq C_{D} \alpha^{-1}\|p\|_{\mathcal{W}}^{2}-\frac{\epsilon}{2}|\boldsymbol{u}|_{a}^{2}-\frac{\alpha^{-1} E_{\max }}{2 \epsilon}\|p\|_{\mathcal{W}}^{2}
\end{aligned}
$$

for any $\epsilon>0$. From (2.11) and using (2.18) and (2.16) gives

$$
\begin{aligned}
\mathcal{B}(\boldsymbol{u}, p, \tilde{p} ; 0,0,-p) & =c(p, p)-d(\tilde{p}, p) \\
& \geq(\alpha \beta)^{-1}|| p \|_{\mathcal{W}}^{2}-|\tilde{p}|_{d}|p|_{d} \\
& \geq(\alpha \beta)^{-1}|| p\left\|_{\mathcal{W}}^{2}-|\tilde{p}|_{d} E_{\max }^{1 / 2}(\alpha \beta)^{-1 / 2}\right\| p \|_{\mathcal{W}} \\
& \geq(\alpha \beta)^{-1}|| p\left\|_{\mathcal{W}}^{2}-\frac{\epsilon_{1}}{2}|\tilde{p}|_{d}^{2}-\frac{(\alpha \beta)^{-1} E_{\max }}{2 \epsilon_{1}}\right\| p \|_{\mathcal{W}}^{2}
\end{aligned}
$$

for any $\epsilon_{1}>0$. We now introduce two parameters $\delta>0$ and $\delta^{\prime}>0$. Combining these two bounds gives

$$
\begin{aligned}
& \mathcal{B}(\boldsymbol{u}, p, \tilde{p} ; \boldsymbol{u}-\left.\delta \boldsymbol{w},-p, \tilde{p}-\delta^{\prime} p\right) \\
&= \mathcal{B}(\boldsymbol{u}, p, \tilde{p} ; \boldsymbol{u},-p, \tilde{p})+\delta \mathcal{B}(\boldsymbol{u}, p, \tilde{p} ;-\boldsymbol{w}, 0,0)+\delta^{\prime} \mathcal{B}(\boldsymbol{u}, p, \tilde{p} ; 0,0,-p) \\
& \geq|\boldsymbol{u}|_{a}^{2}+|\tilde{p}|_{d}^{2}+\delta\left(\frac{1}{\alpha}\left(C_{D}-\frac{E_{\max }}{2 \epsilon}\right)\|p\|_{\mathcal{W}}^{2}-\frac{\epsilon}{2}|\boldsymbol{u}|_{a}^{2}\right) \\
& \quad+\delta^{\prime}\left(\frac{1}{\alpha \beta}\left(1-\frac{E_{\max }}{2 \epsilon_{1}}\right)\|p\|_{\mathcal{W}}^{2}-\frac{\epsilon_{1}}{2}\|\tilde{p}\|_{d}^{2}\right) \\
&=\left(1-\frac{\delta \epsilon}{2}\right)|\boldsymbol{u}|_{a}^{2}+\left(\frac{\delta}{\alpha}\left(C_{D}-\frac{E_{\max }}{2 \epsilon}\right)+\frac{\delta^{\prime}}{\alpha \beta}\left(1-\frac{E_{\max }}{2 \epsilon_{1}}\right)\right)\|p\|_{\mathcal{W}}^{2} \\
& \quad+\left(1-\frac{\delta^{\prime} \epsilon_{1}}{2}\right)|\tilde{p}|_{d}^{2} .
\end{aligned}
$$

Next, making the specific choice

$$
\epsilon=\frac{E_{\max }}{C_{D}}, \quad \delta=\frac{1}{\epsilon}=\frac{C_{D}}{E_{\max }}, \quad \epsilon_{1}=E_{\max }, \quad \delta^{\prime}=\frac{1}{\epsilon_{1}}=\frac{1}{E_{\max }},
$$

and using (2.17) and (2.19) gives

$$
\begin{aligned}
\mathcal{B}(\boldsymbol{u}, p, \tilde{p} ; \boldsymbol{u} & \left.-\delta \boldsymbol{w},-p, \tilde{p}-\delta^{\prime} p\right) \\
& \geq \frac{1}{2}|\boldsymbol{u}|_{a}^{2}+\frac{1}{2}\left(\frac{C_{D}^{2}}{\alpha E_{\max }}+\frac{1}{\alpha \beta E_{\max }}\right)\|p\|_{\mathcal{W}}^{2}+\frac{1}{2}|\tilde{p}|_{d}^{2} \\
& \geq \frac{1}{2} C_{K} E_{\min } \alpha\|\nabla \boldsymbol{u}\|_{\mathcal{W}}^{2}+\frac{1}{2 E_{\max }}\left(\frac{C_{D}^{2}}{\alpha}+\frac{1}{\alpha \beta}\right)\|p\|_{\mathcal{W}}^{2}+\frac{1}{2 \alpha \beta} E_{\min }\|\tilde{p}\|_{\mathcal{W}}^{2} \\
& \geq C\left(\alpha\|\nabla \boldsymbol{u}\|_{\mathcal{W}}^{2}+\left(\frac{1}{\alpha}+\frac{1}{\alpha \beta}\right)\|p\|_{\mathcal{W}}^{2}+\frac{1}{\alpha \beta}\|\tilde{p}\|_{\mathcal{W}}^{2}\right)=: C\|(\boldsymbol{u}, p, \tilde{p})\| \|^{2}
\end{aligned}
$$

where $C=\frac{1}{2} \min \left\{E_{\min } C_{K}, \frac{C_{D}^{2}}{E_{\max }}, \frac{1}{E_{\max }}\right\}$. Since $E_{\min } \leq E_{\max }$ we have shown that (2.22) holds with $\boldsymbol{v}:=\boldsymbol{u}-\delta \boldsymbol{w}, q:=-p, \tilde{q}:=\tilde{p}-\delta^{\prime} p$ with $C \geq E_{\min } C_{1}$ where 
$C_{1}=\frac{1}{2} \min \left\{C_{K}, \frac{C_{D}^{2}}{E_{\max }^{2}}, \frac{1}{E_{\max }^{2}}\right\}$. To complete the proof, we note that

$$
\alpha\|\nabla(\boldsymbol{u}-\delta \boldsymbol{w})\|_{\mathcal{W}}^{2} \leq 2 \alpha\|\nabla \boldsymbol{u}\|_{\mathcal{W}}^{2}+2 \delta^{2} \alpha\|\nabla \boldsymbol{w}\|_{\mathcal{W}}^{2} \leq 2 \alpha\|\nabla \boldsymbol{u}\|_{\mathcal{W}}^{2}+2 \delta^{2} \alpha^{-1}\|p\|_{\mathcal{W}}^{2} .
$$

Similarly,

$$
(\alpha \beta)^{-1}\left\|\tilde{p}-\delta^{\prime} p\right\|_{\mathcal{W}}^{2} \leq 2(\alpha \beta)^{-1}\|\tilde{p}\|_{\mathcal{W}}^{2}+2 \delta^{\prime 2}(\alpha \beta)^{-1}\|p\|_{\mathcal{W}}^{2} .
$$

Using the definition of the norm ||$|\cdot|||$ then leads to the upper bound

$$
\begin{aligned}
\| \mid(\boldsymbol{u}-\delta \boldsymbol{w}, & \left.-p, \tilde{p}-\delta^{\prime} p\right)\|\|^{2} \\
& =\alpha\|\nabla(\boldsymbol{u}-\delta \boldsymbol{w})\|_{\mathcal{W}}^{2}+\left(\frac{1}{\alpha}+\frac{1}{\alpha \beta}\right)\|p\|_{\mathcal{W}}^{2}+\frac{1}{\alpha \beta}\left\|\tilde{p}-\delta^{\prime} p\right\|_{\mathcal{W}}^{2} \\
& \leq\left(2+2 \delta^{2}+2 \delta^{\prime 2}\right)\left(\alpha\|\nabla \boldsymbol{u}\|_{\mathcal{W}}^{2}+\left(\frac{1}{\alpha}+\frac{1}{\alpha \beta}\right)\|p\|_{\mathcal{W}}^{2}+\frac{1}{\alpha \beta}\|\tilde{p}\|_{\mathcal{W}}^{2}\right) \\
& =C_{2}^{2}\|(\boldsymbol{u}, p, \tilde{p})\| \|^{2}
\end{aligned}
$$

as required.

The following theorem is an immediate consequence.

TheOREm 2.4. Given that E satisfies condition (2.1) in Assumption 2.1 the threefield formulation (2.12) admits a unique solution $(\boldsymbol{u}, p, \tilde{p}) \in \mathcal{V} \times \mathcal{W} \times \mathcal{W}$. Moreover,

$$
\|(\boldsymbol{u}, p, \tilde{p}) \mid\| \leq \frac{C_{3}}{E_{\min }} \alpha^{-1 / 2}\|\boldsymbol{f}\|_{L^{2}(D)}
$$

where $C_{3}$ depends on $E_{\max }, C_{K}$ and $C_{D}$.

Proof. Lemma 2.3 ensures that

$$
C_{1} E_{\min }|\|(\boldsymbol{u}, p, \tilde{p})\||^{2} \leq \mathcal{B}(\boldsymbol{u}, p, \tilde{p} ; \boldsymbol{v}, q, \tilde{q})=f(\boldsymbol{v})
$$

where $(\boldsymbol{v}, q, \tilde{q})$ satisfies $\||(\boldsymbol{v}, q, \tilde{q})|\| \leq C_{2}|\|(\boldsymbol{u}, p, \tilde{p})\||$. Applying Cauchy-Schwarz to the right-hand side then gives

$$
\begin{aligned}
\left.C_{1} E_{\min }\||(\boldsymbol{u}, p, \tilde{p})|\|\right|^{2} & \leq \alpha^{-1 / 2}\|\boldsymbol{f}\|_{L^{2}(D)} \alpha^{1 / 2}\|\boldsymbol{v}\|_{L^{2}\left(\Gamma, L^{2}(D)\right)} \\
& \leq \alpha^{-1 / 2}\|\boldsymbol{f}\|_{L^{2}(D)} L \mid\|(\boldsymbol{v}, q, \tilde{q})\| \| \\
& \leq \alpha^{-1 / 2}\|\boldsymbol{f}\|_{L^{2}(D)} L C_{2}\||(\boldsymbol{u}, p, \tilde{p})|\|,
\end{aligned}
$$

where $L$ is the Poincaré-Friedrichs constant associated with $D$. This implies (2.24) with $C_{3}:=L C_{2} / C_{1}$. प

3. Finite-dimensional formulation. To construct an SGFEM approximation of (2.4) we need to introduce a conforming finite element space

$$
V_{h}=\operatorname{span}\left\{\phi_{1}(\boldsymbol{x}), \phi_{2}(\boldsymbol{x}), \ldots, \phi_{n_{u}}(\boldsymbol{x})\right\} \subset H_{E_{0}}^{1}(D),
$$

and then define $\boldsymbol{V}_{h}$ to be the space of vector-valued functions whose components are in $V_{h}$. We will also require a compatible finite element space

$$
W_{h}=\operatorname{span}\left\{\varphi_{1}(\boldsymbol{x}), \varphi_{2}(\boldsymbol{x}), \ldots, \varphi_{n_{p}}(\boldsymbol{x})\right\} \subset L^{2}(D),
$$

in the sense that a discrete inf-sup condition

$$
\sup _{0 \neq \boldsymbol{v} \in \boldsymbol{V}_{h}} \frac{\int_{D} q \nabla \cdot \boldsymbol{v}}{\|\nabla \boldsymbol{v}\|_{\boldsymbol{L}^{2}(D)}} \geq \gamma\|q\|_{L^{2}(D)} \quad \forall q \in W_{h}
$$


is satisfied with $\gamma$ uniformly bounded away from zero (that is, independent of the mesh parameter $h$ ). Two specific inf-sup stable approximation pairs are included in our IFISS software [6] and thus have been extensively tested. These are $\boldsymbol{Q}_{2}-Q_{1}$ (continuous biquadratic approximation for the displacement and continuous bilinear approximation for the pressure) and $\boldsymbol{Q}_{2}-P_{-1}$ (continuous biquadratic approximation for the displacement and discontinuous linear approximation for the pressure) approximations for $\boldsymbol{V}_{h}$ and $W_{h}$ defined on a rectangular element subdivision. ${ }^{1}$

Turning to the parametric discretisation, let $\left\{\psi_{i}\left(y_{j}\right), i=0,1, \ldots\right\}$ denote the set of Legendre polynomials on $\Gamma_{j}$, where $\psi_{i}$ has degree $i$. We fix $\psi_{0}=1$ and assume that the polynomials are normalised in the $L_{\pi_{j}}^{2}\left(\Gamma_{j}\right)$-sense, so that $\left\langle\psi_{i}, \psi_{k}\right\rangle_{\pi_{j}}=\delta_{i, k}$. Next, we choose a set of multi-indices $\Lambda \subset \mathbb{N}_{0}^{M}$ and define the set of multivariate polynomials

$$
S_{\Lambda}:=\operatorname{span}\left\{\psi_{\boldsymbol{\alpha}}(\boldsymbol{y})=\prod_{i=1}^{M} \psi_{\alpha_{i}}\left(y_{i}\right), \quad \boldsymbol{\alpha} \in \Lambda\right\} \subset L_{\pi}^{2}(\Gamma) .
$$

By construction, since $\pi$ is a product measure, the basis functions for $S_{\Lambda}$ are orthonormal with respect to the $L_{\pi}^{2}(\Gamma)$ inner product. We denote $\operatorname{dim}\left(S_{\Lambda}\right)=|\Lambda|=n_{y}$. For instance, if we choose $\Lambda=\left\{\boldsymbol{\alpha}=\left(\alpha_{1}, \ldots, \alpha_{M}\right),|\boldsymbol{\alpha}| \leq p\right\}$, then $n_{y}=\frac{(M+p) !}{M ! p !}$ and $S_{\Lambda}$ contains multivariate polynomials of total degree $p$ or less.

The finite-dimensional version of the three-field problem (2.4) is therefore: find $\left(\boldsymbol{u}_{h, \Lambda}, p_{h, \Lambda}, \tilde{p}_{h, \Lambda}\right) \in \boldsymbol{V}_{h, \Lambda} \times W_{h, \Lambda} \times W_{h, \Lambda}$ such that

$$
\begin{aligned}
a\left(\boldsymbol{u}_{h, \Lambda}, \boldsymbol{v}\right)+b\left(\boldsymbol{v}, p_{h, \Lambda}\right) & =f(\boldsymbol{v}) & & \forall \boldsymbol{v} \in \boldsymbol{V}_{h, \Lambda}, \\
b\left(\boldsymbol{u}_{h, \Lambda}, q\right)-c\left(\tilde{p}_{h, \Lambda}, q\right) & =0 & & \forall q \in W_{h, \Lambda}, \\
-c\left(p_{h, \Lambda}, \tilde{q}\right)+d\left(\tilde{p}_{h, \Lambda}, \tilde{q}\right) & =0 & & \forall \tilde{q} \in W_{h, \Lambda},
\end{aligned}
$$

where we define $\boldsymbol{V}_{h, \Lambda}:=\boldsymbol{V}_{h} \otimes S_{\Lambda}$ and $W_{h, \Lambda}:=W_{h} \otimes S_{\Lambda}$.

The well-posedness of the discrete formulation follows from the stability estimate in Lemma 2.3 together with the discrete inf-sup condition (3.1).

Lemma 3.1. Assuming that $E$ satisfies (2.1) and that the approximation pair $\boldsymbol{V}_{h}, W_{h}$ is inf-sup stable, problem (3.3) admits a unique solution $\left(\boldsymbol{u}_{h, \Lambda}, p_{h, \Lambda}, \tilde{p}_{h, \Lambda}\right) \in$ $\boldsymbol{V}_{h, \Lambda} \times W_{h, \Lambda}, \times W_{h, \Lambda}$ satisfying

$$
\left\|\mid\left(\boldsymbol{u}_{h, \Lambda}, p_{h, \Lambda}, \tilde{p}_{h, \Lambda}\right)\right\| \leq \frac{C_{5}}{E_{\min }} \alpha^{-1 / 2}\|\boldsymbol{f}\|_{L^{2}(D)},
$$

where $C_{5}$ depends on $E_{\max }, C_{K}$ and $\gamma$.

REMARK 3.1. One could, in principle, approximate $p$ and $\tilde{p}$ using different finite element spaces. In this case a second inf-sup condition relating the two pressure spaces would need to be satisfied to ensure a stable approximation overall.

3.1. Linear algebra aspects. We will restrict our attention to planar elasticity from this point onwards. ${ }^{2}$ To formulate the discrete linear system of equations associated with (3.3), a set of sparse matrices and vectors associated with the chosen basis functions for the approximation spaces $V_{h}, W_{h}$ and $S_{\Lambda}$ will need to be assembled. To this end, we first define matrices $G_{0}, G_{k} \in \mathbb{R}^{n_{y} \times n_{y}}$ for $k=1, \ldots, M$, by

$$
\left[G_{0}\right]_{\boldsymbol{\alpha}, \boldsymbol{\beta}}:=\int_{\Gamma} \psi_{\boldsymbol{\alpha}}(\boldsymbol{y}) \psi_{\boldsymbol{\beta}}(\boldsymbol{y}) d \pi(\boldsymbol{y}), \quad\left[G_{k}\right]_{\boldsymbol{\alpha}, \boldsymbol{\beta}}:=\int_{\Gamma} y_{k} \psi_{\boldsymbol{\alpha}}(\boldsymbol{y}) \psi_{\boldsymbol{\beta}}(\boldsymbol{y}) d \pi(\boldsymbol{y}),
$$

\footnotetext{
${ }^{1}$ Both of these mixed approximation strategies are inf-sup stable in a three-dimensional setting.

${ }^{2}$ The extension to three-dimensions is completely straightforward.
} 
where $\boldsymbol{\alpha}, \boldsymbol{\beta} \in \Lambda$. In addition, we define the vector $\boldsymbol{g}_{0} \in \mathbb{R}^{n_{y}}$ to be the first column of $G_{0}$. Since the basis functions for $S_{\Lambda}$ have been chosen to be orthonormal, we have $G_{0}=I$. In addition, due to the three-term recurrence of the underlying univariate families of Legendre polynomials, $G_{k}$ has at most two nonzero entries per row, for each $k=1,2, \ldots, M$, see [14].

We will define the finite element matrix $A_{11}^{k} \in \mathbb{R}^{n_{u} \times n_{u}}$ associated with $V_{h}$ by

$$
\left[A_{11}^{k}\right]_{i, \ell}:=\int_{D} e_{k}(\boldsymbol{x}) \varepsilon\left(\begin{array}{c}
\phi_{i}(\boldsymbol{x}) \\
0
\end{array}\right): \varepsilon\left(\begin{array}{c}
\phi_{\ell}(\boldsymbol{x}) \\
0
\end{array}\right) d \boldsymbol{x}, \quad i, \ell=1, \ldots, n_{u},
$$

for $k=0,1, \ldots, M$ and the matrix $A_{21}^{k} \in \mathbb{R}^{n_{u} \times n_{u}}$ by

$$
\left[A_{21}^{k}\right]_{i, \ell}:=\int_{D} e_{k}(\boldsymbol{x}) \varepsilon\left(\begin{array}{c}
0 \\
\phi_{i}(\boldsymbol{x})
\end{array}\right): \varepsilon\left(\begin{array}{c}
\phi_{\ell}(\boldsymbol{x}) \\
0
\end{array}\right) d \boldsymbol{x}, \quad i, \ell=1, \ldots, n_{u} .
$$

The matrices $A_{12}^{k}, A_{22}^{k} \in \mathbb{R}^{n_{u} \times n_{u}}$ are defined analogously. We can also define matrices $B_{1}, B_{2} \in \mathbb{R}^{n_{p} \times n_{u}}$ so that

$$
\left[B_{1}\right]_{r, \ell}=-\int_{D} \varphi_{r}(\mathbf{x}) \frac{\partial \phi_{\ell}(\mathbf{x})}{\partial x_{1}} d \boldsymbol{x}, \quad\left[B_{2}\right]_{r, \ell}=-\int_{D} \varphi_{r}(\mathbf{x}) \frac{\partial \phi_{\ell}(\mathbf{x})}{\partial x_{2}} d \boldsymbol{x}
$$

for $r=1, \ldots, n_{p}, \ell=1, \ldots, n_{u}$. The mass matrix $C \in \mathbb{R}^{n_{p} \times n_{p}}$ associated with $W_{h}$ is defined by

$$
[C]_{r, s}=\int_{D} \varphi_{r}(\mathbf{x}) \varphi_{s}(\mathbf{x}) d \mathbf{x}, \quad r, s=1, \ldots, n_{p}
$$

and the weighted mass matrices $D_{k} \in \mathbb{R}^{n_{p} \times n_{p}}$ are defined by

$$
\left[D_{k}\right]_{r, s}=\int_{D} e_{k}(\mathbf{x}) \varphi_{r}(\mathbf{x}) \varphi_{s}(\mathbf{x}) d \mathbf{x}, \quad r, s=1, \ldots, n_{p},
$$

for $k=0,1, \ldots, M$. An important point is that if the coefficient $e_{0}(\boldsymbol{x})$ in the expansion of $E$ is a constant then $D_{0}=e_{0} C$. Moreover, if we choose $W_{h}=P_{-1}$ (discontinuous linear pressure approximation) then $C$ is a diagonal matrix and so is $D_{k}$, for each $k=0,1, \ldots, M$. Finally, we define two vectors $\boldsymbol{f}_{1}, \boldsymbol{f}_{2} \in \mathbb{R}^{n_{u}}$ associated with the body force $\boldsymbol{f}(\boldsymbol{x})=\left(f_{1}(\boldsymbol{x}), f_{2}(\boldsymbol{x})\right)^{\top}$, via

$$
\left[\boldsymbol{f}_{1}\right]_{\ell}=\int_{D} f_{1}(\boldsymbol{x}) \phi_{\ell}(\boldsymbol{x}) d \boldsymbol{x}, \quad\left[\boldsymbol{f}_{2}\right]_{\ell}=\int_{D} f_{2}(\boldsymbol{x}) \phi_{\ell}(\boldsymbol{x}) d \boldsymbol{x}, \quad \ell=1, \ldots, n_{u} .
$$

Permuting the variables $p_{h, \Lambda}$ and $\tilde{p}_{h, \Lambda}$ in (3.3) and swapping the order of the second and third equations leads to a saddle-point system of $2\left(n_{u}+n_{p}\right) n_{y}$ equations

$$
\left(\begin{array}{cc}
\mathcal{A} & \mathcal{B}^{\top} \\
\mathcal{B} & 0
\end{array}\right)\left(\begin{array}{l}
\mathbf{v} \\
\mathbf{p}
\end{array}\right)=\left(\begin{array}{l}
\mathbf{b} \\
\mathbf{0}
\end{array}\right)
$$

where $\mathbf{b}_{1}=\mathbf{g}_{0} \otimes \mathbf{f}_{1}, \mathbf{b}_{2}=\mathbf{g}_{0} \otimes \mathbf{f}_{2}$ with vectors

$$
\mathbf{v}=\left(\begin{array}{c}
\mathbf{u} \\
\tilde{\mathbf{p}}
\end{array}\right), \quad \mathbf{b}=\left(\begin{array}{c}
\mathbf{b}_{1} \\
\mathbf{b}_{2}
\end{array}\right)
$$

defined so that $\mathbf{u}, \tilde{\mathbf{p}}$ and $\mathbf{p}$ are the coefficient vectors representing $u_{h, \Lambda}, \tilde{p}_{h, \Lambda}$ and $p_{h, \Lambda}$, respectively in the chosen bases. The coefficient matrix in (3.5) is symmetric with 


$$
\mathcal{A}:=\left(\begin{array}{cc|c}
\alpha \sum_{k=0}^{M} G_{k} \otimes A_{11}^{k} & \alpha \sum_{k=0}^{M} G_{k} \otimes A_{21}^{k} & \mathbf{0} \\
\alpha \sum_{k=0}^{M} G_{k} \otimes A_{12}^{k} & \alpha \sum_{k=0}^{M} G_{k} \otimes A_{22}^{k} & \mathbf{0} \\
\hline \mathbf{0} & \mathbf{0} & (\alpha \beta)^{-1} \sum_{k=0}^{M} G_{k} \otimes D_{k}
\end{array}\right)
$$

and

$$
\mathcal{B}:=\left(\begin{array}{ll}
G_{0} \otimes B_{1} & G_{0} \otimes B_{2} \\
- & -(\alpha \beta)^{-1} G_{0} \otimes C
\end{array}\right) .
$$

Note that due to its very large size, we do not assemble the full coefficient matrix. Operations are only performed via the actions of $G_{0}, G_{k}, A_{11}^{k}, A_{12}^{k}, A_{21}^{k}, A_{22}^{k}, B_{1}, B_{2}$, $C$, and $D_{k}$.

The best way to solve a symmetric saddle-point system iteratively is to use the minimal residual method, see [7, Chapter 4]. Since our system is ill-conditioned, preconditioning is a critical component of our solution strategy.

4. Preconditioning. Following [9], [12], [13] and [17] the most natural preconditioner for the saddle-point system (3.5) is a block preconditioning matrix

$$
P_{\text {approx }}=\left(\begin{array}{cc}
\mathcal{A}_{\text {approx }} & 0 \\
0 & \mathcal{S}_{\text {approx }}
\end{array}\right),
$$

where $\mathcal{A}_{\text {approx }}$ and $\mathcal{S}_{\text {approx }}$ are matrices that are chosen to represent the matrix $\mathcal{A}$ and the Schur complement $\mathcal{S}=\mathcal{B A}^{-1} \mathcal{B}^{\top}$. An important requirement is that the work needed to apply the action of $\mathcal{A}_{\text {approx }}^{-1}$ and $\mathcal{S}_{\text {approx }}^{-1}$ is proportional to the dimension of the associated approximation space.

4.1. Approximation of $\mathcal{A}$. The obvious way to approximate (3.6) is given by

$$
\mathcal{A}_{\text {approx }}=\left(\begin{array}{cc|c}
\alpha G_{0} \otimes A_{11}^{0} & \alpha G_{0} \otimes A_{21}^{0} & \mathbf{0} \\
\alpha G_{0} \otimes A_{12}^{0} & \alpha G_{0} \otimes A_{22}^{0} & \mathbf{0} \\
\hline \mathbf{0} & \mathbf{0} & (\alpha \beta)^{-1}\left(G_{0} \otimes D_{0}\right)
\end{array}\right),
$$

where we denote the diagonal blocks in (4.1) by

$$
\mathcal{A}_{\text {approx }, 1}:=\alpha\left(\begin{array}{cc}
G_{0} \otimes A_{11}^{0} & G_{0} \otimes A_{21}^{0} \\
G_{0} \otimes A_{12}^{0} & G_{0} \otimes A_{22}^{0}
\end{array}\right), \quad \mathcal{A}_{\text {approx }, 2}:=\frac{1}{\alpha \beta}\left(G_{0} \otimes D_{0}\right) .
$$

The fact that $G_{0}=I$ means that the nonzero terms in (4.1) are all block diagonal. Since the finite element matrices $A_{11}^{0}, A_{12}^{0}, A_{21}^{0}, A_{22}^{0}$ and $D_{0}$ all involve the mean coefficient $e_{0}$, we will refer to this strategy as a mean-based approximation. The following lemma quantifies the effectiveness of this approximation.

Lemma 4.1. Let $\mathcal{A}$ and $\mathcal{A}_{\text {approx }}$ be defined in (3.6) and (4.1). If Assumption 2.1 holds, the eigenvalues of $\mathcal{A}_{\text {approx }}^{-1} \mathcal{A}$ lie in the bounded interval $\left[E_{\min } / e_{0}^{\max }, E_{\max } / e_{0}^{\min }\right]$.

Proof. The eigenvalues of $\mathcal{A}_{\text {approx }}^{-1} \mathcal{A}$ can be separated into two distinct sets: each associated with one of the diagonal blocks of $\mathcal{A}$, that is

$$
\mathcal{A}_{1}:=\alpha\left(\begin{array}{cc}
\sum_{k=0}^{M} G_{k} \otimes A_{11}^{k} & \sum_{k=0}^{M} G_{k} \otimes A_{21}^{k} \\
\sum_{k=0}^{M} G_{k} \otimes A_{12}^{k} & \sum_{k=0}^{M} G_{k} \otimes A_{22}^{k}
\end{array}\right), \quad \mathcal{A}_{2}:=\frac{1}{\alpha \beta} \sum_{k=0}^{M} G_{k} \otimes D_{k} .
$$


Let us consider the first block. For any $\boldsymbol{v} \in \mathbb{R}^{2 n_{u} n_{y}}$ there is an associated function $\boldsymbol{r} \in \boldsymbol{V}_{h, \Lambda}$ and using (2.1) and (2.2) gives

$$
\begin{aligned}
\boldsymbol{v}^{\top} \mathcal{A}_{1} \boldsymbol{v}=a(\boldsymbol{r}, \boldsymbol{r}) & =\alpha \int_{\Gamma} \int_{D} E(\boldsymbol{x}, \boldsymbol{y}) \varepsilon(\boldsymbol{r}): \varepsilon(\boldsymbol{r}) d \boldsymbol{x} d \pi(\boldsymbol{y}) \\
& \leq \frac{E_{\max }}{e_{0}^{\min }} \alpha \int_{\Gamma} \int_{D} e_{0}(\boldsymbol{x}) \varepsilon(\boldsymbol{r}): \varepsilon(\boldsymbol{r}) d \boldsymbol{x} d \pi(\boldsymbol{y}) \\
& =\frac{E_{\max }}{e_{0}^{\min }} \boldsymbol{v}^{\top} \mathcal{A}_{\mathrm{approx}, 1} \boldsymbol{v} .
\end{aligned}
$$

Similarly,

$$
\boldsymbol{v}^{\top} \mathcal{A}_{1} \boldsymbol{v} \geq \frac{E_{\min }}{e_{0}^{\max }} \boldsymbol{v}^{\top} \mathcal{A}_{\text {approx }, 1} \boldsymbol{v}
$$

Combining (4.3) and (4.4) gives, for any $\boldsymbol{v} \neq \mathbf{0}$,

$$
\frac{E_{\min }}{e_{0}^{\max }} \leq \frac{\boldsymbol{v}^{\top} \mathcal{A}_{1} \boldsymbol{v}}{\boldsymbol{v}^{\top} \mathcal{A}_{\text {approx }, 1} \boldsymbol{v}} \leq \frac{E_{\max }}{e_{0}^{\min }} .
$$

Let us consider the second block. For any $\boldsymbol{w} \in \mathbb{R}^{n_{p} n_{y}}$ we can define a function $s \in W_{h, \Lambda}$ such that

$$
\begin{aligned}
\boldsymbol{w}^{\top} \mathcal{A}_{2} \boldsymbol{w}=d(s, s) & =(\alpha \beta)^{-1} \int_{\Gamma} \int_{D} E(\boldsymbol{x}, \boldsymbol{y}) s(\boldsymbol{x}, \boldsymbol{y}) s(\boldsymbol{x}, \boldsymbol{y}) d \boldsymbol{x} d \pi(\boldsymbol{y}) \\
\boldsymbol{w}^{\top} \mathcal{A}_{\text {approx }, 2} \boldsymbol{w} & =(\alpha \beta)^{-1} \int_{\Gamma} \int_{D} e_{0}(\boldsymbol{x}) s(\boldsymbol{x}, \boldsymbol{y}) s(\boldsymbol{x}, \boldsymbol{y}) d \boldsymbol{x} d \pi(\boldsymbol{y}) .
\end{aligned}
$$

Making use of (2.1) and (2.2) again gives

$$
\frac{E_{\min }}{e_{0}^{\max }} \leq \frac{\boldsymbol{w}^{\top} \mathcal{A}_{2} \boldsymbol{w}}{\boldsymbol{w}^{\top} \mathcal{A}_{\mathrm{approx}, 2} \boldsymbol{w}} \leq \frac{E_{\max }}{e_{0}^{\min }} .
$$

Combining the bounds for the two Rayleigh quotients completes the proof.

4.2. Refined approximations of $\mathcal{A}$. Inverting the $\mathcal{A}_{\text {approx }, 1}$ block of (4.1) is computationally expensive. To address this we will look for block diagonal alternatives of the form

$$
\tilde{\mathcal{A}}_{\text {approx }, 1}:=\alpha\left(\begin{array}{cc}
G_{0} \otimes \mathbb{A}_{11} & \mathbf{0} \\
\mathbf{0} & G_{0} \otimes \mathbb{A}_{22}
\end{array}\right) .
$$

Herein, we will consider two different choices of $\mathbb{A}_{11}$ and $\mathbb{A}_{22}$. The first option is to take $\mathbb{A}_{11}=\mathbb{A}_{22}=\mathbb{A}:=2\left(A_{11}^{0}+A_{22}^{0}\right) / 3$. Note that, if $e_{0}=1$ then for any $\boldsymbol{v} \in \mathbb{R}^{n_{u}}$ we have $\boldsymbol{v}^{\top} \mathbb{A} \boldsymbol{v}=\left\|\nabla v_{h}\right\|_{L^{2}(D)}^{2}$ where $v_{h}$ is the finite element function in $V_{h}$ represented by $\boldsymbol{v}$. That is, $\mathbb{A}$ gives a discrete representation of the scalar Laplacian operator.

LEMMA 4.2. Let $\mathbb{A}_{11}=\mathbb{A}_{22}=2\left(A_{11}^{0}+A_{22}^{0}\right) / 3$. If Assumption 2.1 holds then all eigenvalues $\sigma_{\mathcal{A}}$ of $\mathcal{A}_{\text {approx }}^{-1} \mathcal{A}$, where $\mathcal{A}_{\text {approx }}$ has leading diagonal block (4.7), satisfy

$$
\sigma_{\mathcal{A}} \in\left[C_{K} \frac{E_{\min }}{e_{0}^{\max }}, \frac{E_{\max }}{e_{0}^{\min }}\right],
$$

where $0<C_{K} \leq 1$ is the Korn constant. 
Proof. For any $\boldsymbol{v} \in \mathbb{R}^{2 n_{u} n_{y}}$, we can define a function $\boldsymbol{r} \in \boldsymbol{V}_{h, \Lambda}$ such that,

$$
\begin{aligned}
\boldsymbol{v}^{\top} \mathcal{A}_{1} \boldsymbol{v} & =\alpha \int_{\Gamma} \int_{D} E(\boldsymbol{x}, \boldsymbol{y}) \varepsilon(\boldsymbol{r}): \boldsymbol{\varepsilon}(\boldsymbol{r}) d \boldsymbol{x} d \pi(\boldsymbol{y}) \\
& \leq E_{\max } \alpha \int_{\Gamma} \int_{D} \nabla \boldsymbol{r}: \nabla \boldsymbol{r} d \boldsymbol{x} d \pi(\boldsymbol{y}) \\
& \leq \frac{E_{\max }}{e_{0}^{\min }} \alpha \int_{\Gamma} \int_{D} e_{0}(\boldsymbol{x}) \nabla \boldsymbol{r}: \nabla \boldsymbol{r} d \boldsymbol{x} d \pi(\boldsymbol{y}) \\
& =\frac{E_{\max }}{e_{0}^{\min }} \boldsymbol{v}^{\top} \tilde{\mathcal{A}}_{\text {approx }, 1} \boldsymbol{v} .
\end{aligned}
$$

Analagously,

$$
\begin{aligned}
\boldsymbol{v}^{\top} \mathcal{A}_{1} \boldsymbol{v} & \geq E_{\min } \alpha \int_{\Gamma} \int_{D} \varepsilon(\boldsymbol{r}): \boldsymbol{\varepsilon}(\boldsymbol{r}) d \boldsymbol{x} d \pi(\boldsymbol{y}) \\
& \geq E_{\min } \alpha C_{K} \int_{\Gamma} \int_{D} \nabla \boldsymbol{r}: \nabla \boldsymbol{r} d \boldsymbol{x} d \pi(\boldsymbol{y}) \\
& \geq \frac{E_{\min }}{e_{0}^{\max }} \alpha C_{K} \int_{\Gamma} \int_{D} e_{0}(\boldsymbol{x}) \nabla \boldsymbol{r}: \nabla \boldsymbol{r} d \boldsymbol{x} d \pi(\boldsymbol{y}) \\
& =\frac{E_{\min }}{e_{0}^{\max }} C_{K} \boldsymbol{v}^{\top} \tilde{\mathcal{A}}_{\text {approx }, 1} \boldsymbol{v} .
\end{aligned}
$$

Combining (4.9) and (4.10) leads to bounds for the Rayleigh quotient

$$
\frac{E_{\min }}{e_{0}^{\max }} C_{K} \leq \frac{\boldsymbol{v}^{\top} \mathcal{A}_{1} \boldsymbol{v}}{\boldsymbol{v}^{\top} \tilde{\mathcal{A}}_{\text {approx }, 1} \boldsymbol{v}} \leq \frac{E_{\max }}{e_{0}^{\min }},
$$

and hence for the eigenvalues of $\tilde{\mathcal{A}}_{\text {approx }, 1}^{-1} \mathcal{A}_{1}$. The bound (4.6) provides a bound for the eigenvalues of $\mathcal{A}_{\text {approx }, 2}^{-1} \mathcal{A}_{2}$. Combining these two bounds gives the stated result.

For our second choice of $\mathbb{A}_{11}$ and $\mathbb{A}_{22}$, we simply discard the off-diagonal blocks of the mean-based approximation $\mathcal{A}_{\text {approx }, 1}$. The strategy will not be pursued here since it results in an inferior eigenvalue bound.

Lemma 4.3. Let $\mathbb{A}_{11}=A_{11}^{0}$ and $\mathbb{A}_{22}=A_{22}^{0}$. If Assumption 2.1 holds then all eigenvalues $\sigma_{\mathcal{A}}$ of $\mathcal{A}_{\text {approx }}^{-1} \mathcal{A}$, where $\mathcal{A}_{\text {approx }}$ has leading diagonal block (4.7), satisfy

$$
\sigma_{\mathcal{A}} \in\left[C_{K} \frac{E_{\min }}{e_{0}^{\max }}, 2 \frac{E_{\max }}{e_{0}^{\min }}\right]
$$

where $0<C_{K} \leq 1$ is the Korn constant.

Proof. The proof is a minor variation of that of Lemma 4.2. By obtaining bounds for both Rayleigh quotients separately, we find that the eigenvalues lie in

$$
\left[C_{K} \frac{E_{\min }}{e_{0}^{\max }}, 2 \frac{E_{\max }}{e_{0}^{\min }}\right] \cup\left[\frac{E_{\min }}{e_{0}^{\max }}, \frac{E_{\max }}{e_{0}^{\min }}\right],
$$

which yields the stated result.

REMARK 4.1. The bounds (4.8) and (4.11) depend on the Young's modulus E and on the Korn constant $C_{K}$ but are independent of all discretisation parameters. 
4.3. Approximation of $\mathcal{S}$. Given a block diagonal approximation to $\mathcal{A}_{1}$ of the form (4.7), an approximation to the Schur complement matrix $\mathcal{S}$ can be constructed so that $\tilde{\mathcal{S}}_{\text {approx }}:=\mathcal{B} \tilde{\mathcal{A}}_{\text {approx }}^{-1} \mathcal{B}^{\top}$. Since this is a dense matrix it is not a practical preconditioner. The next result introduces a sparse block diagonal matrix $P_{\mathcal{S}}$ and establishes that it is spectrally equivalent to $\tilde{\mathcal{S}}_{\text {approx }}$.

LEMma 4.4. Suppose that $\tilde{\mathcal{S}}_{\text {approx }}:=\mathcal{B} \tilde{\mathcal{A}}_{\text {approx }}^{-1} \mathcal{B}^{\top}$ where, in the definition (4.7) we make the choice $\mathbb{A}_{11}=\mathbb{A}_{22}=2\left(A_{11}^{0}+A_{22}^{0}\right) / 3$. Defining

$$
P_{S}:=\left(\alpha^{-1}+(\alpha \beta)^{-1}\right) I \otimes C,
$$

where $C$ is the pressure mass matrix, we have

$$
\theta^{2} \leq \frac{\boldsymbol{w}^{\top} \tilde{\mathcal{S}}_{\text {approx }} \boldsymbol{w}}{\boldsymbol{w}^{\top} P_{\mathcal{S}} \boldsymbol{w}} \leq \Theta^{2} \quad \forall \boldsymbol{w} \in \mathbb{R}^{n_{p} n_{y}}
$$

with $\theta^{2}=\gamma^{2} / e_{0}^{\max }, \Theta^{2}=2 / e_{0}^{\min }$, where $\gamma$ is the discrete inf-sup constant in (3.1) associated with the finite element spaces $\boldsymbol{V}_{h}$ and $W_{h}$.

Proof. Using the definitions of $\mathcal{B}$ and $\tilde{\mathcal{A}}_{\text {approx }}$ and the fact that $G_{0}=I$ gives

$$
\begin{aligned}
\tilde{\mathcal{S}}_{\text {approx }}= & \left(I \otimes B_{1}\right)\left(\alpha\left(I \otimes \mathbb{A}_{11}\right)\right)^{-1}\left(I \otimes B_{1}\right)^{\top}+\left(I \otimes B_{2}\right)\left(\alpha\left(I \otimes \mathbb{A}_{22}\right)\right)^{-1}\left(I \otimes B_{2}\right)^{\top} \\
& +\left(-(\alpha \beta)^{-1} I \otimes C\right)\left((\alpha \beta)^{-1}\left(I \otimes D_{0}\right)\right)^{-1}\left(-(\alpha \beta)^{-1} I \otimes C\right)^{\top} \\
= & \alpha^{-1}\left(I \otimes\left(B_{1} \mathbb{A}_{11}^{-1} B_{1}^{\top}\right)+I \otimes\left(B_{2} \mathbb{A}_{22}^{-1} B_{2}^{\top}\right)\right)+(\alpha \beta)^{-1}\left(I \otimes C D_{0}^{-1} C^{\top}\right) \\
= & \alpha^{-1}(I \otimes X)+(\alpha \beta)^{-1}\left(I \otimes C D_{0}^{-1} C^{\top}\right),
\end{aligned}
$$

where $X:=\left(B_{1} \mathbb{A}_{11}^{-1} B_{1}^{\top}+B_{2} \mathbb{A}_{22}^{-1} B_{2}^{\top}\right)$.

The fact that the matrices $\mathbb{A}_{11}$ and $\mathbb{A}_{22}$ represent discrete Laplacian operators weighted by the mean field $e_{0}$, gives the matrix $X$ a structure that can be exploited. Specifically we can combine the bounds in [7, Proposition 3.24] with the bounds on $e_{0}$ in (2.2) to give a two-sided bound

$$
\frac{\gamma^{2}}{e_{0}^{\max }} \leq \frac{\boldsymbol{w}^{\top}(I \otimes X) \boldsymbol{w}}{\boldsymbol{w}^{\top}(I \otimes C) \boldsymbol{w}} \leq \frac{2}{e_{0}^{\min }} \quad \forall \boldsymbol{w} \in \mathbb{R}^{n_{p} n_{y}},
$$

where $\gamma$ is the inf-sup constant (as defined in (3.1)). We also have a two-sided bound for the two component mass matrices

$$
e_{0}^{\min } C \leq D_{0} \leq e_{0}^{\max } C,
$$

where the inequalities hold entrywise. Combining these results with (4.14) gives

$$
\begin{aligned}
\boldsymbol{w}^{\top} \tilde{\mathcal{S}}_{\text {approx }} \boldsymbol{w} & =\alpha^{-1} \boldsymbol{w}^{\top}(I \otimes X) \boldsymbol{w}+(\alpha \beta)^{-1} \boldsymbol{w}^{\top}\left(I \otimes C D_{0}^{-1} C^{\top}\right) \boldsymbol{w}, \\
& \leq 2\left(\alpha e_{0}^{\min }\right)^{-1} \boldsymbol{w}^{\top}(I \otimes C) \boldsymbol{w}+\left(\alpha \beta e_{0}^{\min }\right)^{-1} \boldsymbol{w}^{\top}(I \otimes C) \boldsymbol{w}, \\
& \leq 2\left(e_{0}^{\min }\right)^{-1} \boldsymbol{w}^{\top} P_{\mathcal{S}} \boldsymbol{w} .
\end{aligned}
$$

Similarly,

$$
\begin{gathered}
\boldsymbol{w}^{\top} \tilde{\mathcal{S}}_{\text {approx }} \boldsymbol{w} \geq \\
=\left(\alpha e_{0}^{\max }\right)^{-1} \gamma^{2} \boldsymbol{w}^{\top}(I \otimes C) \boldsymbol{w}+\left(\alpha \beta e_{0}^{\max }\right)^{-1} \boldsymbol{w}^{\top} I \otimes C \boldsymbol{w}, \\
=\gamma^{2}\left(e_{0}^{\max }\right)^{-1} \boldsymbol{w}^{\top} P_{\mathcal{S}} \boldsymbol{w}
\end{gathered}
$$


Combining the upper and lower bounds leads to the stated result. $\mathrm{\square}$

REMARK 4.2. In a practical setting, the mean field $e_{0}$ in (1.2) is often taken to be constant. In this case we could define $P_{\mathcal{S}}:=\left(\alpha^{-1}+(\alpha \beta)^{-1}\right) e_{0}^{-1} I \otimes C$ and get a refined estimate

$$
\theta^{2}:=\gamma^{2} \leq \frac{\boldsymbol{w}^{\top} \tilde{\mathcal{S}}_{\text {approx }} \boldsymbol{w}}{\boldsymbol{w}^{\top} P_{\mathcal{S}} \boldsymbol{w}} \leq 2:=\Theta^{2} \quad \forall \boldsymbol{w} \in \mathbb{R}^{n_{p} n_{y}} .
$$

Notice that $P_{S}$ is block diagonal but if we choose $W_{h}=P_{-1}$ then $C$ is diagonal and hence so is $P_{S}$.

We will summarise our preferred methodology at this point: the preconditioner of choice is a block diagonal matrix

$$
\mathcal{P}:=\left(\begin{array}{ccc}
\tilde{\mathcal{A}}_{\text {approx }, 1} & 0 & 0 \\
0 & \mathcal{A}_{\text {approx }, 2} & 0 \\
0 & 0 & P_{\mathcal{S}}
\end{array}\right),
$$

where $\tilde{\mathcal{A}}_{\text {approx }, 1}$ is as defined in (4.7) with $\mathbb{A}_{11}=\mathbb{A}_{22}=2\left(A_{11}^{0}+A_{22}^{0}\right) / 3, \mathcal{A}_{\text {approx }, 2}$ is defined in (4.2) and $P_{S}$ is defined in (4.12) (or else as in (4.17) if $e_{0}$ is constant).

We note that each of the three diagonal blocks of the preconditioner, $\tilde{\mathcal{A}}_{\text {approx }, 1}$, $\mathcal{A}_{\text {approx }, 2}$ and $P_{\mathcal{S}}$ provides a discrete representation of a norm that is equivalent to one of the terms in the norm (2.21). This strategy is consistent with the preconditioning philosophy of Mardal \& Winther [13] and ensures that the eigenvalues of the preconditioned system can be bounded independently of the discretisation parameters. This is formally expressed in the following concluding result.

THEOREM 4.5. Let $\mu_{\min }$ and $\mu_{\max }$ be the extremal eigenvalues of $\mathcal{A}_{\text {approx }}^{-1} \mathcal{A}$, where $\mathcal{A}_{\text {approx }}$ has leading diagonal block (4.7) with $\mathbb{A}_{11}=\mathbb{A}_{22}=2\left(A_{11}^{0}+A_{22}^{0}\right) / 3$. Then the eigenvalues of

$$
\mathcal{P}^{-1 / 2}\left(\begin{array}{cc}
\mathcal{A} & \mathcal{B}^{\top} \\
\mathcal{B} & 0
\end{array}\right) \mathcal{P}^{-1 / 2}
$$

lie in the union of the intervals

$$
\begin{aligned}
& {\left[\frac{1}{2}\left(\mu_{\min }-\sqrt{\mu_{\min }^{2}+4 \Theta^{2}}\right)\right.}\left., \frac{1}{2}\left(\mu_{\max }-\sqrt{\mu_{\max }^{2}+4 \theta^{2}}\right)\right] \\
& \cup\left[\mu_{\min }, \frac{1}{2}\left(\mu_{\max }^{2}+\sqrt{\mu_{\max }+4 \Theta^{2}}\right)\right],
\end{aligned}
$$

where the constants $\theta$ and $\Theta$ are given in Lemma 4.4 if $P_{\mathcal{S}}$ is as defined in (4.12), or else are given in (4.17) if $P_{\mathcal{S}}$ has the alternative definition given in Remark 4.2.

Proof. The proof follows from Lemma 2.1 of [16] and Corollary 3.4 of [15].

Recall that bounds for the eigenvalues of $\tilde{\mathcal{A}}_{\text {approx }}^{-1} \mathcal{A}$ are given in (4.8). Hence, the bounds for the eigenvalues for the preconditioned system depend only on the discrete inf-sup constant $\gamma$ in (3.1), the Korn constant $C_{K}$ and the ratios $E_{\min } / e_{0}^{\max }$ and $E_{\max } / e_{0}^{\min }$. Note that the eigenvalue bounds are robust in the incompressible limit. A direct consequence of our eigenvalue bound is that the number of MINRES iterations needed to converge to a fixed tolerance when solving the Galerkin system is guaranteed to be bounded by a constant that is independent of all discretisation parameters as well as the Poisson ratio. This will be illustrated by numerical results in the final section. 
5. Numerical results. In this section we consider a representative test problem taken from the S-IFISS toolbox [3] and we study the practical performance of the block diagonal preconditioning strategy that was analysed above. The spatial domain is $D=(-1,1) \times(-1,1)$. We impose a homogeneous Neumann boundary condition on the right edge $\partial D_{N}=\{1\} \times(-1,1)$ and a zero essential boundary condition for the displacement on $\partial D_{D}=\partial D \backslash \partial D_{N}$. The body force is chosen to be $\boldsymbol{f}=(1,1)^{\top}$. The Young's modulus has constant mean value one and takes the form

$$
E(\boldsymbol{x}, \boldsymbol{y})=1+\sigma \sqrt{3} \sum_{m=1}^{M} \sqrt{\lambda_{m}} \varphi_{m}(\boldsymbol{x}) y_{m},
$$

where $\sigma$ is the standard deviation and $\left\{\left(\lambda_{m}, \varphi_{m}\right)\right\}$ are the eigenpairs of the integral operator associated with $\left(1 / \sigma^{2}\right) C\left(\boldsymbol{x}, \boldsymbol{x}^{\prime}\right)$, where

$$
C\left(\boldsymbol{x}, \boldsymbol{x}^{\prime}\right)=\sigma^{2} \exp \left(-\frac{1}{2}\left\|\boldsymbol{x}-\boldsymbol{x}^{\prime}\right\|_{1}\right), \quad \boldsymbol{x}, \boldsymbol{x}^{\prime} \in D .
$$

For the spatial approximation, we use $Q_{2}-P_{-1}-P_{-1}$ mixed finite elements. That is, continuous biquadratic approximation for the displacement and discontinuous linear approximation for both of the Lagrange multipliers. In this case, the approximation $P_{\mathcal{S}}$ to the Schur complement is a diagonal matrix. For the parametric approximation, we choose $S_{\Lambda}$ to be the set of polynomials of total degree $p$ or less in $y_{1}, \ldots, y_{M}$ on $\Gamma=[-1,1]^{M}$. In Table 5.1 we record the number of spatial degrees of freedom associated with the finite element discretisation (as the refinement level $\ell$ is varied) and in Table 5.2 we record the dimension of the space $S_{\Lambda}$ (when $M$ and $p$ are varied). Recall that the number of equations to be solved is $2\left(n_{u}+n_{p}\right) n_{y}$. For example, when we have $M=10$ input parameters, the grid level is set to $\ell=6$ and the polynomial degree is $p=4$, we have over fourteen million equations to solve.

TABLE 5.1

Number of deterministic degrees of freedom associated with $\boldsymbol{Q}_{2}-P_{-1}-P_{-1}$ approximation.

\begin{tabular}{|c|c|c|c|}
\hline \multicolumn{4}{|c|}{ deterministic degrees of freedom } \\
\hline Refinement-level $(l)$ & $n_{u}$ & $n_{p}$ & $2\left(n_{u}+n_{p}\right)$ \\
\hline 4 & 240 & 192 & 864 \\
5 & 992 & 768 & 3,520 \\
6 & 4,032 & 3,072 & 14,208 \\
\hline
\end{tabular}

TABLE 5.2

Number of parametric degrees of freedom associated with the chosen multi-index set $\Lambda$.

\begin{tabular}{|c|c|c|c|}
\hline \multicolumn{4}{|c|}{$n_{y}$} \\
\hline$p$ & $M=5$ & $M=8$ & $M=10$ \\
\hline 3 & 56 & 165 & 286 \\
4 & 126 & 495 & 1,001 \\
\hline
\end{tabular}

We examine the eigenvalues of the preconditioned SGFEM system first. The est_minres code that is built into S-IFISS exploits the connection with the Lanczos algorithm (see [7, section 2.4]) and generates accurate harmonic Ritz values estimates of the underlying eigenvalue spectrum as the preconditioned system is being solved. 
Details are given in Silvester \& Simoncini [17]. The extremal eigenvalue estimates are computed on the fly and are reproduced in Tables 5.3 and $5.4 .^{3}$ We consider two values of the Poisson ratio $\nu$, and two values for the standard deviation $\sigma$ (values which guarantee that all realisations of $E$ are positive) and vary $M$ and $\ell$. The polynomial degree $p=3$ is fixed. We observe that the widths of the intervals containing the estimated eigenvalues are independent of the spatial discretisation parameter as well as the number $M$ of parameters. While the intervals are slightly wider for $\nu=0.49999$ than for $\nu=0.4$, they are bounded as $\nu \rightarrow 1 / 2$. The interior eigenvalue bounds are closer to the origin, however, for the larger value of $\sigma$. The price we pay for using a mean-based preconditioner (which is by definition block diagonal) is that the resulting eigenvalue bounds depend on the ratios $E_{\min } / e_{0}^{\max }$ and $E_{\max } / e_{0}^{\min }$. In this example, these quantities depend on $\sigma$. However, we stress that $\sigma$ cannot be chosen arbitrarily large. Assumption 2.1 must be satisfied, otherwise the problem is not well posed. Preconditioning schemes that are not mean-based may lead to more tightly clustered eigenvalues but in general are not as computationally efficient.

TABLE 5.3

Bound for eigenvalues of preconditioned SGFEM system, $\sigma=0.085, p=3$.

\begin{tabular}{|c|c|c|}
\hline \multicolumn{3}{|c|}{ Computed eigenvalue } \\
\hline \multicolumn{2}{|c|}{$l=5$} \\
\hline$M$ & $\nu=.4$ & $\nu=.49999$ \\
\hline 5 & {$[-0.8287,-0.3369] \cup[0.2737,1.8332]$} & {$[-0.9347,-0.1892] \cup[0.2878,1.8886]$} \\
8 & {$[-0.8305,-0.3368] \cup[0.2722,1.8408]$} & {$[-0.9058,-0.1891] \cup[0.2859,1.8934]$} \\
10 & {$[-0.8311,-0.3367] \cup[0.2720,1.8427]$} & {$[-0.9064,-0.1891] \cup[0.2857,1.8949]$} \\
\hline \multicolumn{2}{|c|}{$l=6$} \\
\hline 5 & {$[-0.8291,-0.3368] \cup[0.2731,1.8358]$} & {$[-0.9047,-0.1890] \cup[0.2866,1.8910]$} \\
8 & {$[-0.8323,-0.3366] \cup[0.2715,1.8448]$} & {$[-0.9084,-0.1890] \cup[0.2849,1.8986]$} \\
10 & {$[-0.8334,-0.3366] \cup[0.2713,1.8469]$} & {$[-0.9094,-0.1890] \cup[0.2848,1.9006]$} \\
\hline
\end{tabular}

TABLE 5.4

Bound for eigenvalues of preconditioned SGFEM system, $\sigma=0.17, p=3$.

\begin{tabular}{|c|c|c|}
\hline \multicolumn{3}{|c|}{ Computed eigenvalue } \\
\hline \multicolumn{3}{|c|}{$l=5$} \\
\hline$M$ & $\nu=.4$ & $\nu=.49999$ \\
\hline 5 & {$[-0.9291,-0.3178] \cup[0.2318,1.9435]$} & {$[-0.9491,-0.1789] \cup[0.2428,1.9935]$} \\
8 & {$[-0.8797,-0.3171] \cup[0.2268,1.9566]$} & {$[-0.9538,-0.1789] \cup[0.2358,2.0052]$} \\
10 & {$[-0.8817,-0.3169] \cup[0.2264,1.9604]$} & {$[-0.9555,-0.1788] \cup[0.2352,2.0086]$} \\
\hline \multicolumn{3}{|c|}{$l=6$} \\
\hline 5 & {$[-0.9206,-0.3176] \cup[0.2307,1.9454]$} & {$[-0.9507,-0.1787] \cup[0.2413,1.9964]$} \\
8 & {$[-0.8836,-0.3167] \cup[0.2254,1.9623]$} & {$[-0.9581,-0.1787] \cup[0.2346,2.0126]$} \\
10 & {$[-0.8857,-0.3166] \cup[0.2251,1.9663]$} & {$[-0.9600,-0.1785] \cup[0.2336,2.0167]$} \\
\hline
\end{tabular}

In Table 5.5 we record the number of MINRES iterations required to reduce the preconditioned residual error to $10^{-6}$ for the case $\sigma=0.085$, with $p=3$ fixed and varying $M$ and $\ell$. In Tables 5.6 and 5.7 we record the number of iterations required

\footnotetext{
${ }^{3}$ The associated MINRES relative residual tolerance is set to $10^{-6}$. Bounds are unchanged if we rerun the experiments with a tighter tolerance.
} 
when $\sigma=0.17$ with $p=3$ and $p=4$ fixed, respectively. The timings were recorded running S-IFISS on a MacBook Pro with $16 \mathrm{~Gb}$ of memory and a $2.3 \mathrm{GHz}$ Intel Core i5 processor. We observe that for a fixed value of $\sigma$, the iteration counts remain stable as the discretisation parameters $\ell$ and $p$ are varied. Moreover, the iteration counts stay bounded when working with values of $\nu$ arbitrarily close to $1 / 2$.

TABLE 5.5

MINRES iteration counts for stopping tolerance $10^{-6}$, and timings in seconds (in parentheses), $\sigma=0.085, p=3$.

\begin{tabular}{|l|l|l|l|l|l|}
\hline$M$ & $\nu=.4$ & $\nu=.49$ & $\nu=.499$ & $\nu=.4999$ & $\nu=.49999$ \\
\hline \multicolumn{5}{|c|}{$l=5$} \\
\hline 5 & $56(3.9)$ & $74(5.3)$ & $78(5.7)$ & $78(5.7)$ & $78(5.8)$ \\
8 & $56(10)$ & $75(12.8)$ & $78(13.7)$ & $79(13.7)$ & $79(13.4)$ \\
10 & $56(16.5)$ & $75(22.5)$ & $79(23.6)$ & $79(23.4)$ & $79(23.5)$ \\
\hline \multicolumn{5}{|c|}{$l=6$} \\
\hline 5 & $56(14.6)$ & $75(19.7)$ & $79(20.9)$ & $79(20.5)$ & $79(20.9)$ \\
8 & $56(45.2)$ & $75(60.5)$ & $79(64.2)$ & $79(63.8)$ & $79(64)$ \\
10 & $56(86)$ & $75(114.3)$ & $79(120.5)$ & $79(118.1)$ & $79(117.3)$ \\
\hline
\end{tabular}

TABLE 5.6

MINRES iteration counts for stopping tolerance $10^{-6}$, and timings in seconds (in parentheses), $\sigma=0.17, p=3$.

\begin{tabular}{|l|l|l|l|l|l|}
\hline$M$ & $\nu=.4$ & $\nu=.49$ & $\nu=.499$ & $\nu=.4999$ & $\nu=.49999$ \\
\hline \multicolumn{5}{|c|}{$l=5$} \\
\hline 5 & $66(5.4)$ & $86(6.3)$ & $90(6.8)$ & $92(6.9)$ & $92(6.9)$ \\
8 & $67(11.5)$ & $88(15.4)$ & $92(15.8)$ & $93(16.4)$ & $93(15.9)$ \\
10 & $67(19.9)$ & $88(27)$ & $93(28.4)$ & $93(28.1)$ & $93(28.6)$ \\
\hline \multicolumn{5}{|c|}{$l=6$} \\
\hline 5 & $66(18.3)$ & $88(24.4)$ & $92(25.5)$ & $92(25.5)$ & $92(25.5)$ \\
8 & $67(55.4)$ & $88(70.7)$ & $93(75.8)$ & $93(75.4)$ & $93(76.6)$ \\
10 & $67(102.4)$ & $89(134.9)$ & $93(140.4)$ & $95(145)$ & $95(142)$ \\
\hline
\end{tabular}

TABLE 5.7

MINRES iteration counts for stopping tolerance $10^{-6}$, and timings in seconds (in parentheses), $\sigma=0.17, p=4$.

\begin{tabular}{|l|l|l|l|l|l|}
\hline$M$ & $\nu=.4$ & $\nu=.49$ & $\nu=.499$ & $\nu=.4999$ & $\nu=.49999$ \\
\hline \multicolumn{5}{|c|}{$l=5$} \\
\hline 5 & $67(8.1)$ & $90(11.6)$ & $95(12)$ & $95(12)$ & $95(12)$ \\
8 & $70(34.4)$ & $93(44.4)$ & $97(45.6)$ & $98(48.9)$ & $98(48.1)$ \\
10 & $70(69.4)$ & $93(94.1)$ & $98(96.7)$ & $98(96.5)$ & $98(94.2)$ \\
\hline \multicolumn{5}{|c|}{$l=6$} \\
\hline 5 & $69(39.9)$ & $91(50.6)$ & $95(54.7)$ & $96(54.6)$ & $96(53.6)$ \\
8 & $70(176.8)$ & $94(233.3)$ & $98(249.4)$ & $98(249.2)$ & $98(250.6)$ \\
10 & $70(378.2)$ & $94(513.9)$ & $98(538.1)$ & $98(538.7)$ & $98(534.3)$ \\
\hline
\end{tabular}


6. Conclusions. This work analyses parameter-robust discretizations and the construction of preconditioners for linear elasticity problems with uncertain material parameters. Having introduced a new three-field formulation of the problem, it is rigorously shown that preconditioners that are based on mapping properties associated with a specific parameter-dependent norm are robust with respect to variations of the Poisson ratio, the choice of finite elements spaces as well as the discretization parameters. The theoretical results are confirmed by a systematically designed set of numerical experiments. There are several generalizable aspects of our work. The idea of introducing an additional auxiliary variable to avoid working with parameterdependent coefficients that exhibit rational nonlinearities is applicable to other PDE problems. The ideas underpinning the construction of the block diagonal preconditioner apply to other PDEs with parameter-dependent coefficients where stochastic Galerkin approximation leads to a discrete problem with the structure (3.5). Finally, the preconditioning methodology gives a starting point for designing efficient solution algorithms for more challenging (e.g., nonlinear) elasticity models with uncertain material coefficients.

\section{REFERENCES}

[1] I. Babuška, R. Tempone, And G. E. Zouraris, Galerkin finite element approximations of stochastic elliptic partial differential equations, SIAM Journal on Numerical Analysis, 42 (2004), pp. 800-825.

[2] A. Bespalov, C. E. Powell, and D. J. Silvester, A priori error analysis of stochastic Galerkin mixed approximations of elliptic PDEs with random data, SIAM Journal on Numerical Analysis, 50 (2012), pp. 2039-2063.

[3] A. Bespalov, D. J. Silvester, and C. E. Powell, Stochastic IFISS (S-IFISS) version 1.04, June 2017. http://www.manchester.ac.uk/ifiss/s-ifiss1.0.tar.gz.

[4] S. Brenner and R. Scott, The mathematical theory of finite element methods, vol. 15, Springer Science \& Business Media, 2007.

[5] M. Eigel, C. J. Gittelson, C. Schwab, And E. Zander, Adaptive stochastic Galerkin FEM, Computer Methods in Applied Mechanics and Engineering, 270 (2014), pp. 247-269.

[6] H. Elman, A. Ramage, And D. Silvester, IFISS: A computational laboratory for investigating incompressible flow problems, SIAM Review, 56 (2014), pp. 261-273. http://dx.doi.org/10.1137/120891393.

[7] H. Elman, D. Silvester, And A. Wathen, Finite Elements and Fast Iterative Solvers: with Applications in Incompressible Fluid Dynamics, Oxford University Press, Oxford, UK, 2014. Second Edition, xiv+400 pp. ISBN: 978-0-19-967880-8.

[8] A. Ern and J.-L. Guermond, Theory and Practice of Finite Elements, Springer, New York, 2004.

[9] O. G. Ernst, C. E. Powell, D. J. Silvester, and E. Ullmann, Efficient solvers for a linear stochastic Galerkin mixed formulation of diffusion problems with random data, SIAM Journal on Scientific Computing, 31 (2009), pp. 1424-1447.

[10] L. R. Herrmann, Elasticity equations for incompressible and nearly incompressible materials by a variational theorem, AIAA J., 3 (1965), pp. 1896-1900.

[11] A. Khan, C. E. Powell, And D. J. Silvester, Robust a posteriori error estimators for mixed approximation of nearly incompressible elasticity, arXiv e-prints, (2017). https://arxiv.org/abs/1710.03328.

[12] A. KlaWonn, An optimal preconditioner for a class of saddle point problems with a penalty term, SIAM Journal on Scientific Computing, 19 (1998), pp. 540-552.

[13] K.-A. Mardal and R. Winther, Preconditioning discretizations of systems of partial differential equations, Numerical Linear Algebra with Applications, 18 (2011), pp. 1-40.

[14] C. E. Powell and H. C. Elman, Block-diagonal preconditioning for spectral stochastic finiteelement systems, IMA Journal of Numerical Analysis, 29 (2009), pp. 350-375.

[15] C. E. Powell and D. J. Silvester, Optimal preconditioning for Raviart-Thomas mixed formulation of second-order elliptic problems, SIAM Journal on Matrix Analysis and Applications, 25 (2003), pp. 718-738.

[16] T. Rusten and R. Winther, A preconditioned iterative method for saddlepoint problems, 
SIAM Journal on Matrix Analysis and Applications, 13 (1992), pp. 887-904.

[17] D. Silvester And V. Simoncini, An optimal iterative solver for symmetric indefinite systems stemming from mixed approximation, ACM Transactions on Mathematical Software, 37 (2011). http://dx.doi.org/10.1145/1916461.1916466. 